\title{
Centro Universitario UTEG a través de la consultoría universitaria fortalece las finanzas de la microempresa en la zona metropolitana de Guadalajara. UTEG University Center University consulting through strengthened the finances of microenterprise in the Guadalajara metropolitan área. Centro Universitário UTEG através de consultoria faculdade reforça financiamento de microempresas na área metropolitana de Guadalajara
}

\author{
Miguel Ángel Haro Ruiz \\ Centro Universitario UTEG, México \\ miguel.haro@uteg.edu.mx \\ Carmen Adriana Barjas Sánchez \\ Centro Universitario UTEG, México \\ cbarajass@uteg.edu.mx \\ Maria Candelaria Dolores Luquin Topete \\ Centro Universitario UTEG, México \\ maria.luquin@uteg.edu.mx
}

\section{Resumen}

La microempresa establecida en la zona metropolitana de Guadalajara, México, se caracteriza como unidad económica dirigida por una sola persona, es quien toma las decisiones principales de la administración, organización, dirección, control de sus recursos financieros, la mayoría de las ocasiones estás se realizan de manera empírica, derivado de lo anterior el Centro Universitario UTEG, a través del área de investigación y la dirección académica de la Licenciatura en Contaduría Pública, diseño un programa de consultoría empresarial universitaria que permitiera acercar a los estudiantes, docentes e investigadores con los empresarios con la finalidad de ofrecerles capacitación y actualización permanente de acuerdo a las necesidades y debilidades particulares detectadas en el diagnostico FODA de cada microempresa consultada, con el objetivo de fortalecer sus finanzas mediante la 
implementación de un plan de acción a corto, mediano y plazo, atendiendo áreas funcionales como la contabilidad, las finanzas y las obligaciones fiscales, de esta manera su ciclo de vida se extiende y unidad económica se consolida, crece y desarrolla.

El modelo de la consultoría empresarial universitaria utilizado en la presente investigación facilita la interacción y sensibilización del empresario con los estudiantes, docentes y profesionales que aportan sus conocimientos y experiencia profesional, permitiéndoles intervenir de manera inmediata en la solución de las necesidades y debilidades encontradas en cada microempresa.

Palabras clave: Consultoría, microempresa, finanzas.

\section{Abstract}

Microenterprises established in metropolitan Guadalajara, Mexico, is characterized as directed by one economic unit, it is who makes the decisions of major management, organisation management, control of its financial resources, the majority of cases are held in an empirical manner, derived from the above UTEG University Center, through research and academic degree in public accounting from direction, I design a program of University business consulting that would bring together students, teachers and researchers with entrepreneurs in order to provide them with training and continual updating according to the needs and particular weaknesses detected in the Diagnostics SWOT of each microenterprise consulted, in order to strengthen their finances through the implementation of a plan of action in the short medium and long-term, functional areas such as accounting, finance and tax obligations, in this way of life extends and economic unit is consolidated, grows and develops.

University business consulting model used in this research facilitates interaction and awareness of the entrepreneur with the students, teachers and professionals who bring their knowledge and professional experience, allowing them to intervene immediately in the solution of needs and weaknesses found in each micro-enterprise. 
Key words: consultancy, micro enterprise, finance.

\section{Resumo}

Microempresa estabelecida na área metropolitana de Guadalajara, México, caracteriza-se como uma unidade económica liderado por uma pessoa, é quem faz as grandes decisões da administração, organização, gestão, controle de seus recursos financeiros, a maioria são, por vezes, fez empiricamente derivado do acima do Centro universitário UTEG, através do domínio da investigação e da direção acadêmica do Curso de Licenciatura em Contabilidade, conceber um programa de consultoria de negócios universidade que traria estudantes, professores e pesquisadores com empresários, a fim de proporcionar formação e actualização de acordo com as necessidades particulares e fracos identificados no diagnóstico SWOT de cada microempresa consultado, a fim de fortalecer as suas finanças através da implementação de um plano de acção a curto, médio e longo participando de áreas funcionais, como obrigações de contabilidade, finanças e impostos, assim que seu ciclo de vida se estende e consolida unidade económica, cresce e se desenvolve.

O modelo de consultoria de negócios universidade utilizado nesta pesquisa facilita a interação e conscientização empresário com alunos, professores e profissionais que contribuem com seu conhecimento e experiência profissional, permitindo-lhes intervir imediatamente em atender as necessidades e os pontos fracos encontrados em cada microempresa.

Palavras-chave: Consulting, finanças microempresa.

Fecha recepción: Octubre 2015

Fecha aceptación: Mayo 2016 


\section{Introducción}

El Centro Universitario UTEG, a través del investigador, la Dirección Académica y el equipo de docentes de la Licenciatura en Contaduría Pública, diseñaron e implementaron un modelo de consultoría empresarial universitaria, con la finalidad de propiciar la interacción y sensibilización de los empresarios con los estudiantes, docentes y profesionales de la Contaduría Pública, con el objetivo primordial de capacitarlos y actualizarlos en los diferentes temas contables, fiscales y áreas funcionales de las organizaciones que les permita tomar decisiones fundamentadas y además basadas en estados financieros básicos que les ayuden a elegir las opciones pertinentes que favorezcan el fortalecimiento de las finanzas de la unidad económica por microempresa que esta sea.

El Plan de Desarrollo Institucional 2025 (PDI) del Centro Universitario UTEG, establece un marco de referencia qué guía cada una de las acciones y procesos administrativos, académicos, de investigación, de vinculación y extensión que impacten significativamente en cada una de las actividades que se desarrollen dentro y fuera de UTEG, con la finalidad de facilitar la interacción con los sectores productivos y sociales, entre los que destacan los siguientes objetivos y planes de acción que permiten alcanzarlos de manera precisa a través de la vinculación socialmente responsable, destacando las siguientes acciones y objetivos:

Función: Implementar acciones articuladas que construyan y mantengan un puente permanente de intercambio de recursos.

Información y servicios entre el Centro Universitario UTEG y su entorno; para asegurar que las funciones sustantivas de la institución contribuyan al desarrollo de los sectores social, privado y público.

El objetivo establecido en el PDI, que incide de manera directa para lograr lo consecución del programa de consultoría empresarial universitaria es el siguiente:

Objetivo 4.1.

Implementar un proceso de vinculación sensible y efectivo de respuesta a las problemáticas sociales.

Estrategias

4.1.1 Focalizar problemáticas sociales a atender en relación a las líneas de investigación. 
4.1.2 Establecer convenios institucionales con los grupos de interés: (Organizaciones no gubernamentales (ONG), asociaciones de profesionistas, colonos, gobierno, empresas, entre otros).

4.1.3 Difundir en los medios masivos de comunicación y redes sociales las acciones de vinculación desarrolladas.

Con la finalidad de ofrecer servicios académicos de calidad y que estos se vinculen de manera eficiente, así como impulsar el talento emprendedor que contribuya al desarrollo de las Micro, Pequeñas y Medianas Empresas (MIPYMES), se creó el Centro de Gestión Empresarial, con el propósito de generar y desarrollar nuevos proyectos empresariales y hacer crecer a las entidades económicas ya existentes, a través de una asesoría y consultoría empresarial universitaria con estándares de calidad y especializadas en temas de administración financiera, contabilidad, costos, finanzas, impuestos y auditoría entre otros.

El Centro Universitario UTEG busca fortalecer las finanzas de las microempresas del sector comercio, mediante la colaboración efectiva entre el Centro de Gestión Empresarial y las diferentes direcciones académicas de los programas educativos de las ciencias económicas y administrativas, destacando algunos proyectos exitosos como:

a) Un despacho de asesoría contable atendido por docentes y estudiantes que prestan su servicio social y sus prácticas profesionales de la carrera de contaduría pública, actualmente proporcionan asesoría contable, financiera y fiscal alrededor de sesenta contribuyentes.

b) Fue la primer Universidad en contar con un Módulo de atención a contribuyentes a nivel estatal, y que opera de manera conjunta con el Servicio de Administración Tributaria (SAT), el cual debido a su buenas prácticas fue elegido entre varias de su tipo, y observado para reproducir en el modelo EUROSOCIAL en otros países, su forma de operar al realizar actividades de extensión, vinculación, prácticas y servicio social con docentes responsables, personal del SAT y alumnos de la carrera de contaduría pública, por sus excelentes resultados. 
c) Convenio de colaboración académica, de servicio social y prácticas profesionales con el Colegio de Contadores Públicos de Guadalajara, Jalisco A.C. el cuál ha permitido incorporar a estudiantes de la Licenciatura en Contaduría Pública de UTEG a realizar actividades académicas vinculadas con las diferentes áreas disciplinarias como la contable, la financiera, la de costos y la fiscal, de esta manera interactúan directamente con profesionales de la carrera y con los tomadores de decisiones como lo son los empresarios, traduciéndose dichas actividades en consultorías empresariales en escenarios reales que les han permitido enriquecer sus competencias disciplinares y profesionales in situ y de acuerdo a las propias necesidades de las diferentes organizaciones ya sean estas públicas o privadas.

La consultoría empresarial universitaria, no es una actividad nueva en México, mucho menos en el resto del mundo, sin embargo está, se puede perfeccionar en la manera que las IES, incidan significativamente a través de las acciones académicas y productos de investigación surgidos en las aulas, mediante la interacción permanente de los estudiantes y docentes. La presente propuesta parte desde su marco teórico y las propuestas de diferentes autores en dicho tema, con la intención de rescatar los casos de éxito, que permitan diseñar una consultoría eficaz y de incidencia inmediata que detone el fortalecimiento de las finanzas de las microempresas del sector comercio ubicadas en Guadalajara, Jalisco, México.

Es importante resaltar la importancia de las MYPYMES de acuerdo a la estratificación establecida por la Secretaría de Economia del Gobierno Federal Mexicano en el año 2009, quedando la clasificación de acuerdo a los siguientes criterios: 
Se establece la estratificación de las MiPyME, de conformidad con los siguientes criterios:

Figura No. 1 Estratificación MiPyME

\begin{tabular}{|c|c|c|c|c|}
\hline \multicolumn{5}{|c|}{ Estratificación } \\
\hline Tamaño & Sector & $\begin{array}{l}\text { Rango de número } \\
\text { de trabajadores }\end{array}$ & $\begin{array}{l}\text { Rango de monto de } \\
\text { ventas anuales } \\
\text { (mdp) }\end{array}$ & $\begin{array}{l}\text { Tope máximo } \\
\text { combinado* }\end{array}$ \\
\hline Micro & Todas & Hasta 10 & Hasta \$4 & 4.6 \\
\hline \multirow[t]{2}{*}{ Pequeña } & Comercio & Desde 11 hasta 30 & $\begin{array}{l}\text { Desde \$4.01 hasta } \\
\$ 100\end{array}$ & 93 \\
\hline & $\begin{array}{l}\text { Industria } \quad \text { y } \\
\text { Servicios }\end{array}$ & Desde 11 hasta 50 & $\begin{array}{l}\text { Desde } \$ 4.01 \text { hasta } \\
\$ 100\end{array}$ & 95 \\
\hline \multirow{3}{*}{ Mediana } & Comercio & Desde 31 hasta 100 & \multirow[b]{2}{*}{$\begin{array}{l}\text { Desde } \$ 100.01 \\
\text { hasta } \$ 250\end{array}$} & \multirow[b]{2}{*}{235} \\
\hline & Servicios & Desde 51 hasta 100 & & \\
\hline & Industria & Desde 51 hasta 250 & $\begin{array}{l}\text { Desde } \$ 100.01 \\
\text { hasta } \$ 250\end{array}$ & 250 \\
\hline
\end{tabular}

Fuente: Ley para el Desarrollo de la Competitividad en México, de la Micro, Pequeña y Mediana Empresa, publicada en el DOF, el día 30 de junio del 2009.

Éste tipo de empresas son la base de la economía mexicana, ha provocado un incremento en la generación de empleos así como la aportación del más del 50\% del PIB en México, de ahí la importancia que tienen en el país, el de crear acciones que permitan sostener y que se consoliden dichas MYPYMES.

Respecto a la denominación de las MIPYMES, existen distintas definiciones en el resto del mundo, esto debido a que, en cada país tienen su propia definición de acuerdo a criterios particulares y la tipología utilizada para conceptualizarlas. En México las MIPYMES constituyen un sector fundamental para el desarrollo económico, a nivel nacional representan alrededor del 99 por ciento del total de las unidades económicas, además aportan en promedio el 52 por ciento del Producto Interno Bruto (PIB) del país, con la generación de más del 70 por ciento de los empleos formales, todo esto de acuerdo a datos 
oficiales emitidos y publicados en su página de internet, por la Secretaría de Economía (SE).

De acuerdo a la exposición de motivos de la reforma fiscal a la Ley del Impuesto Sobre la Renta (LISR) para el año 2017, enviada por el Presidente de México a través del Secretario de Hacienda a la mesa directiva de la Cámara de Diputados el pasado 8 de Septiembre de 2016, menciona que de acuerdo con la información del último censo económico del año 2014 publicado por el Instituto Nacional de Estadística y Geografía (INEGI), el 97.4\% de las empresas establecidas en México son microempresas, y que estas a su vez generan el $50 \%$ de empleo en el país, de acuerdo a estos datos oficiales es pertinente que las Instituciones de Educacion Superior (IES) públicas o privadas, así como los gobiernos federal, estatal y municipal a través de la triple hélice, construyan vínculos estratégicos y efectivos que permitan fortalecer las finanzas de las microempresas así como sus áreas funcionales que les facilite desarrollarse en el ámbito empresarial, local, regional, nacional e internacional.

El modelo de la consultoría empresarial universitaria es una estrategia que contribuye al desarrollo y crecimiento de la microempresa y además permite a los estudiantes interactuar y explotar sus conocimientos teóricos y técnicos en escenarios reales a través de la solución

de problemas y casos que viven día a día las empresas, es pertinente, precisar como la Ley para el Desarrollo de la Competitividad en México, de la Micro, Pequeña y Mediana Empresa, publicada en el DOF, el día 30 de junio del 2009, define de manera textual y literal a la "Consultoría".

Consultoría: "Servicio empresarial que consiste en la transferencia de conocimientos, metodología y aplicaciones, con la finalidad de mejorar los procesos de la empresa que reciben la atención"

La consultoría empresarial, es un proceso que potencializa las diferentes áreas funcionales de las microempresas, es necesario identificar varias definiciones de autores que han desarrollado investigaciones relacionadas con el perfeccionamiento de cada una de las etapas de la consultoría. 
(Kubr, 2015), describe dos enfoques que facilitan la interpretación de la consultoría empresarial, desde una visión funcional, apoyándose con la definición de Fritz Steele, el cual establece: "cualquier forma de proporcionar ayuda sobre el contenido, proceso o estructura de una tarea o de un conjunto de tareas, en que el consultor no es efectivamente responsable de la ejecución de la tarea misma, sino que ayuda a los que lo son", esto quiere decir, que el consultor guía cada una de las acciones que tienen que desarrollar los actores principales y que toman decisiones en una entidad económica, involucrando a todos los colaboradores sin importar el nivel de responsabilidad en el que se encuentren.

Además (Kubr, 2015), menciona que la consultoría de empresas puede enfocarse como un servicio profesional o como un método de prestar asesoramiento y ayuda prácticos, en otras palabras; la asesoría tiene que realizarse de manera fundamentada sobre diagnósticos, estados financieros, análisis de puestos, entre otros, y estas deben de involucrar acciones individuales y colaborativas de las áreas involucradas con la finalidad de coadyuvar a la organización a solucionar y resolver los problemas y necesidades encontrados en los diferentes instrumentos utilizados por los profesionales que llevan a cabo la consultoría empresarial.

Según (Hernández, 2011), la consultoría debe ser un programa de formación eficiente, esté genera un aumento en la productividad, mejora las relaciones interpersonales y además eleva el nivel de la calidad en todas sus áreas funcionales, lo que provoca cambios cuantitativos que se hacen evidentes inmediatamente en cada una de las acciones y decisiones del empresarios y colaboradores de la unidad económica.

También enlista algunos aspectos que debe cumplir el profesional que lleva a cabo el rol de consultor, entre los que destacan los siguientes:

- Debe crear un clima de apertura, un ambiente de confianza y respecto a la individualidad y capacidad de las personas.

- Debe provocar el trabajo colaborativo y el logro de metas comunes.

- Debe favorece a la solución de problemas individuales y en equipo, mediante el análisis de las causas y de esta manera generar opciones de solución. 
- Debe proponer instrumentos que faciliten la realización de acciones y el cumplimiento de los objetivos y metas planteados.

- Debe sensibilizar a los participantes en la consultoría con la finalidad de resolver los problemas y necesidades detectados.

- Debe de supervisar e intervenir de manera oportuna cada una de las acciones que realizan los involucrados en la consultoría.

La consultoría empresarial universitaria, debe sistematizar cada una las acciones realizadas en las áreas administrativas y financieras de manera tal, que estas decisiones se realicen de manera conjunta entre expertos consultores y empresarios, con el apoyo de los estudiantes de las diferentes carreras de las ciencias económicas y administrativas que se ofrecen en el Centro Universitario UTEG, con la intención de precisar teóricamente la importancia que tiene la consultoría. Está se debe entender como una serie de asesorías fundamentadas a través de estudios y análisis teóricos, científicos de las áreas funcionales de las entidades económicas, así como en distintas normas jurídicas, contables y fiscales como lo son:

El área administrativa, legal, contable, de costos, de recursos humanos, mercadotecnia, fiscal y dónde repercute la toma de decisiones: el área financiera.

La finalidad de la consultoría empresarial es ofrecer al empresario o comerciante, asesorías empresariales integrales de calidad y profesionales, que les permita tomar decisiones acertadas y fundamentadas que coadyuven a lograr los objetivos y metas planteadas, debido a que, en el crecimiento, desarrollo y consolidación de negocios depende de varias aristas internas, externas, nacionales e internacionales que son difíciles de predecir y a través de la consultoría empresarial universitaria, se pueden atender de manera inmediata y asertiva.

(Hernández, 2011), define al proceso como una red de actividades vinculadas ordenadamente, y que se llevan a cabo de manera reiterativa mediante la utilización de recursos e información que les permite transformar insumos en productos, abarca desde el inicio hasta la satisfacción de las necesidades del cliente. 
La consultoría empresarial se concibe como un proceso que permite identificar, analizar e intervenir de manera directa e indirecta a través de actividades colaborativas fundamentadas en teorías, principios, normas y leyes que facilitan la solución de los problemas, las necesidades y áreas de oportunidad de la organización con la finalidad de implementar un plan de mejora continua a través de capacitación y actualización permanente de todos los actores que intervienen en la toma de decisiones con el propósito de asegurar la competitividad de la empresa u organización.

Lo anterior permite fundamentar la metodología empleada en el proyecto de consultoría empresarial universitaria implementado en el UTEG, cuya propuesta es fortalecer las finanzas de las microempresas del sector comercio de la zona metropolitana de la ciudad de Guadalajara, Jalisco. México. Se identificó la oportunidad en la cual el Centro Universitario UTEG, tiene la posibilidad de vincular de manera efectiva las actividades académicas, científicas y profesionales que se realizan de manera natural en el proceso de enseñanza aprendizaje entre administrativos, docentes, expertos y estudiantes de las diversas carreras de las ciencias económicas y administrativas.

Antes de iniciar con la implementación de la consultoría empresarial universitaria, el cuerpo colegiado diseño un proceso de reclutamiento, selección y capacitación de estudiantes de $7^{\circ}$ y $8^{\circ}$ semestre de la Licenciatura en Contaduría Pública, con la finalidad de formarlos con las competencias profesionales y disciplinares básicas en el ámbito de los negocios y además capacitarlos en la metodología del proceso de la consultoría empresarial.

La capacitación a docentes y alumnos se centrara principalmente en la sensibilización del programa de consultoría empresarial universitaria, y en los temas del diseño, aplicación e interpretación del instrumento FODA en las áreas funcionales de las empresas: proceso administrativo, sistema contable, análisis financiero, obligaciones fiscales, políticas de ventas, crédito y cobranza, la formulación de proyectos de inversión y sus elementos básicos, el diseño de un plan de negocios, entre otros. 
Una vez que se capaciten a los estudiantes de las diversas carreras, se conformaran equipos de trabajo disciplinares integrado por un docente experto el cual fungirá como consultor líder de cada equipo, con la finalidad de sensibilizarlos de la importancia que tiene vincular los aprendizajes adquiridos y desarrollados en las aulas a través del proceso de la consultoría empresarial universitaria, en la cual van a tener la oportunidad de implementar acciones vivenciales y practicas reales de sus conocimientos en diferentes contextos y escenarios de su vida laboral y profesional, con la guía y asesoría del consultor líder.

El éxito de la consultoría empresarial también radica a través del trabajo en equipo, (Furnham, 2001) lo define como grupos formales que están constituidos por personas encargadas de tomar decisiones con la finalidad de lograr un objetivo específico, tienen derechos y obligaciones que cumplir de manera individual y colaborativa, el desempeño eficiente de cada uno de los integrantes del equipo de trabajo contribuyen en gran medida en el desarrollo de las diferentes actividades independientemente del rol que asuman en el proceso de la consultoría empresarial universitaria.

\section{Metodología utilizada}

El proceso de la consultoría empresarial debe de ser cíclico y permanente con una evaluación de los resultados que le permita corregir a tiempo los errores o debilidades detectados en las diferentes etapas y momentos, la finalidad es fortalecer las finanzas de las microempresas a corto, mediano y largo plazo, con la intención de consolidar el crecimiento y expansión de este tipos de empresas, las cuales en la ciudad de Guadalajara, Jalisco. México, tienen una representación de hasta el 99 por ciento del sector comercio local, según datos del Instituto de Información Estadística y Geografía el estado de Jalisco, México (IIEG), así como su incidencia en la estabilidad económica y social es trascendente.

(Kubr, 2015) establece una serie de fases que en su conjunto forman el proceso de la consultoría que según él debe realizarse, de acuerdo a las etapas siguientes: 
1.- Iniciación

- Primeros contactos con el cliente

- Diagnostico preliminar de los problemas

- Planificación del cometido

- Propuestas de tareas al cliente

- Contrato de Consultoría

2.- Diagnóstico

- Análisis del objetivo

- Análisis del problema

- Descubrimiento de los hechos

- Análisis y síntesis de los hechos

- Información de los resultados del cliente

3.- Planificación de medidas

- Elaboración de soluciones

- Evaluación de opiniones

- Propuestas al cliente

- Planificación de la aplicación de medidas

4.- Aplicación

- Contribuir a la aplicación

- Propuestas de ajustes

- Capacitación

5.- Terminación

- Evaluación

- Informe final

- Establecimiento de compromisos

- Planes de seguimiento 
- Retirada

Las cinco fases anteriores muestran y guían de manera clara los diferentes momentos y actividades que conlleva realizar un proceso de consultoría, para el caso del programa de consultoría empresarial universitaria diseñado y aplicado en el Centro Universitario UTEG, incorporamos algunas fases que permiten lograr los objetivos planteados por el equipo de consultores y el PDI de la propia institución educativa, además se enriquecieron las diferentes etapas de la consultoría mediante la experiencia profesional de los docentes e investigadores, además de aprovechar las decisiones que toman día a día los empresarios y que les han favorecido para consolidar su negocio.

Para la implementación de la metodología empleada en el proceso de la consultoría empresarial universitaria por el equipo de consultores, se estableció un cronograma de actividades que facilitara la consecución de las actividades y los objetivos planteados en el programa, quedando de la siguiente manera: 
Figura No. 2 Cronograma de actividades

\begin{tabular}{|c|c|c|c|}
\hline Actividad & Fecha & Participantes & Resultados \\
\hline $\begin{array}{l}\text { Propuesta, justificación } \\
\text { y pre diagnóstico a cada } \\
\text { una de las empresas } \\
\text { consultadas. }\end{array}$ & $\begin{array}{l}\text { Abril a Noviembre } \\
2015\end{array}$ & $\begin{array}{l}\text { Dr. Miguel Ángel Haro Ruiz } \\
\text { Dra. Rocío García Romero } \\
\text { Mtra. Carmen Álvarez } \\
\text { Esquivel } \\
\text { Mtra. Carmen Adriana Barajas } \\
\text { Sánchez } \\
\text { Mtra. Maricela Álvarez } \\
\text { Mtro. Alfonso Ortiz Sandate } \\
\text { L.C.P. José Miguel Ramírez } \\
\text { Muro }\end{array}$ & $\begin{array}{l}\text { Programa de consultoría } \\
\text { empresarial universitaria } \\
\text { aprobado. } \\
\begin{array}{l}\text { Diseño del Diagnostico } \\
\text { FODA }\end{array}\end{array}$ \\
\hline $\begin{array}{l}\text { Presentación del } \\
\text { programa y } \\
\text { sensibilización } \\
\text { (Secretaría General y } \\
\text { Dirección Académica de } \\
\text { LCP) }\end{array}$ & $\begin{array}{l}\text { Diciembre } 2015 \text { a } \\
\text { Enero } 2016\end{array}$ & $\begin{array}{l}\text { Dr. Miguel Ángel Haro Ruiz } \\
\text { Dra. Rocío García Romero } \\
\text { Mtra. Carmen Álvarez Esquivel } \\
\text { Mtra. Carmen Adriana Barajas } \\
\text { Sánchez } \\
\text { Mtro. Alfonso Ortiz Sandate } \\
\text { Mtro. Francisco Zuno } \\
\text { Mtra. Maricela Álvarez } \\
\text { Esquivel } \\
\text { L.C.P. José Miguel Ramírez } \\
\text { Muro }\end{array}$ & $\begin{array}{l}\text { Integración y } \\
\text { sensibilización de los } \\
\text { docentes de la carrera al } \\
\text { programa de consultoría } \\
\text { empresarial universitaria }\end{array}$ \\
\hline $\begin{array}{l}\text { Invitación a empresarios } \\
\text { a incorporarse al } \\
\text { proyecto de vinculación } \\
\text { empresarial. }\end{array}$ & Enero 2016 & $\begin{array}{l}\text { Dr. Miguel Ángel Haro Ruiz } \\
\text { Dra. Rocío García Romero } \\
\text { Empresarias (AMMJE) } \\
\text { Jóvenes Empresarias de Jalisco } \\
\text { Empresas (Despacho } \\
\text { Contable) } \\
\text { Hecho por Mujeres (Gobierno } \\
\text { Municipal de Guadalajara) }\end{array}$ & $\begin{array}{l}\text { Invitación y sensibilización } \\
\text { a } 60 \text { empresarios para } \\
\text { incorporarse al programa } \\
\text { de consultoría empresarial } \\
\text { universitaria. }\end{array}$ \\
\hline $\begin{array}{l}\text { Capacitación } \\
\text { formación a Directores } \\
\text { Académicos, } \\
\text { Empresarios } \\
\text { Estudiantes }\end{array}$ & $\begin{array}{l}\text { Febrero a Junio } \\
2016\end{array}$ & $\begin{array}{l}\text { Directores, Académicos, } \\
\text { Empresarios y Estudiantes }\end{array}$ & 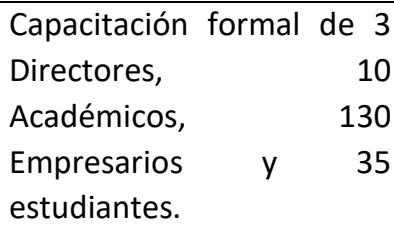 \\
\hline $\begin{array}{l}\text { Integración de equipos } \\
\text { de trabajo de la carrera } \\
\text { de Contaduría Pública, } \\
\text { Empresarios } \\
\text { Académicos. }\end{array}$ & Marzo 2016 & $\begin{array}{l}\text { Estudiantes de las carreras de } \\
\text { Contaduría Pública, Director } \\
\text { Académico, Empresarios, } \\
\text { Académicos y Estudiantes. }\end{array}$ & $\begin{array}{l}\text { Conformación de } 10 \\
\text { equipos de trabajo para } \\
\text { llevar a cabo el proceso de } \\
\text { la consultoría empresarial } \\
\text { universitaria. }\end{array}$ \\
\hline $\begin{array}{l}\text { Pre diagnóstico y } \\
\text { análisis de resultados }\end{array}$ & Abril 2016 & $\begin{array}{l}\text { Director Académico } \\
\text { Académicos } \\
\text { Estudiantes } \\
\text { Empresarios }\end{array}$ & $\begin{array}{l}\text { Aplicación y análisis de } 15 \\
\text { diagnósticos FODA }\end{array}$ \\
\hline $\begin{array}{l}\text { Implementación del } \\
\text { plan de acción de la } \\
\text { consultoría empresarial }\end{array}$ & $\begin{array}{l}\text { Mayo a Junio } \\
2016\end{array}$ & $\begin{array}{l}\text { Director Académico } \\
\text { Académicos } \\
\text { Estudiantes } \\
\text { Empresarios }\end{array}$ & $\begin{array}{l}\text { Plan de acción por cada } \\
\text { una de las } 15 \text { empresas } \\
\text { consultadas }\end{array}$ \\
\hline
\end{tabular}




\begin{tabular}{|lr|lr|l|l|}
\hline $\begin{array}{l}\text { Informe final del } \\
\text { proceso Julio y Agosto } \\
\text { consultoría empresarial }\end{array}$ & $\begin{array}{l}\text { Director Académico } \\
\text { Académicos } \\
\text { Estudiantes } \\
\text { Empresarios }\end{array}$ & $\begin{array}{l}\text { Informe final y plan de } \\
\text { negocios de 15 empresas } \\
\text { consultadas. }\end{array}$ \\
\hline
\end{tabular}

La propuesta del pre diagnostico FODA está integrada en una de las fases del proceso de la consultoría empresarial universitaria, estas se tienen que desarrollar en un plazo de seis a ocho meses y deben ser cíclicas hasta alcanzar las metas establecidas por el empresario.

\section{1.- Entrevista Inicial}

Contempla la entrevista del consultor líder con el empresario, con la intención de identificar las características comerciales, legales y fiscales de la entidad económica a través de un diagnostico preliminar.

Se identifican los resultados del diagnóstico preliminar se presenta la propuesta de tareas y actividades a desarrollar de manera conjunta con los estudiantes, consultor y empresarios, mediante la formalización de un acuerdo confidencial.

Formalización del contrato para iniciar el proceso de la consultoría empresarial entre el o los empresarios y el equipo consultor.

\section{2.- Aplicación del diagnóstico FODA}

Se realiza la aplicación del diagnóstico FODA de las áreas administrativa, contable, de costos, de mercadotecnia, fiscal y financiera de la entidad económica, a través de un instrumento que permita valorar los resultados de una manera cualitativa y cuantitativa.

Se evalúan los resultados obtenidos de cada una de las áreas diagnosticadas de la entidad económica con la finalidad de diseñar un plan de trabajo que atienda de manera oportuna cada uno de los indicadores.

\section{3.- Diseño conjunto del plan de acción}

El equipo consultor diseña el plan de trabajo de cada una de las actividades que se tienen que desarrollar para atender de manera oportuna el indicador y las características de la entidad económica, se elabora una propuesta del plan de cada una de las acciones a desarrollar en tiempo y forma al empresario. 
Se socializa de manera conjunta y detallada el plan de acción entre empresario, consultor y estudiantes, se acuerdan las acciones y medidas que se van a implementar atendiendo los resultados obtenidos y características propias de la entidad económica.

\section{4.- Implementación del plan de acción}

El equipo consultor de manera conjunta con el empresario realiza cada una de las acciones y actividades acordadas en tiempo y forma como lo establecieron en el plan de acción.

De manera permanente se supervisan cada una de las acciones implementadas en el proceso del plan de acción, observando los resultados obtenidos y socializándolos y sensibilizando a todos los actores de la consultoría.

\section{5.- Evaluación y seguimiento}

La evaluación de los resultados de cada una de las acciones, actividades, compromisos y estrategias implementadas en el plan de acción, se tiene que realizar de manera permanente y oportuna, con la finalidad de detectar omisiones o errores y en su caso resultados no esperados.

En la etapa de la evaluación es importante identificar las áreas de oportunidad, con el propósito de resolver las necesidades o problemáticas detectadas con un plan de intervención inmediata a través de estrategias de capacitación, asesoría oportuna y toma de decisiones que modifiquen la acción implementada.

\section{6.- Reflexión e intervención cíclica}

Es la etapa que permite reflexionar e intervenir de manera permanente sobre los resultados obtenidos de cada uno de los compromisos, acciones y actividades implementadas en las diferentes áreas de la entidad económica y en las diversas etapas del proceso de la consultoría empresarial universitaria, con la finalidad de continuar con un proceso de mejora continua que permita fortalecer las finanzas de las MIPYMES a corto, mediano y largo plazo. 
El diseño de la metodología implementado en el programa de consultoría empresarial universitaria de UTEG, es flexible y permite en cualquier momento intervenir en cada una de las diferentes etapas, con la finalidad de contribuir de manera conjunta con los empresarios en la toma de decisiones que fortalezcan las áreas funcionales de su empresa u organización, una vez establecida la metodología a aplicar, el equipo de consultores tuvo acercamiento con varias asociaciones de empresarios, entre los que destacan la Asociación Mexicana de Mujeres Jóvenes Empresarias, capitulo Zapopan (AMMJE), Caja Popular Mexicana sucursales de la zona metropolitana de Guadalajara, y el programa de hecho por mujeres auspiciado por el gobierno municipal de Guadalajara periodo 2015-2017, resultado de dichas gestiones se logró sensibilizar a varios empresarios, de los cuales treinta de más de cincuenta aceptaron incorporarse al proyecto de consultoría empresarial universitaria y a su vez los participantes experimentan diferentes acciones y estrategias que les permiten acceder a información relevante para llevarla a cabo, en la primera fase (Entrevista inicial), participan los siguientes actores:

- 30 Empresarios del sector comercio.

- 10 Consultores-docentes (Contadores y Abogados).

- 40 Estudiantes de la licenciatura en Contaduría Pública

- 6 Expertos del área de Gestión Empresarial y Consultorías Universitarias.

Es importante mencionar que fue difícil el acercamiento, así como el proceso de sensibilización y capacitación con los organismos y organizaciones antes mencionados, a pesar de tener varias reuniones de trabajo y manifestarles que el programa de consultoría empresarial es gratuito no causo interés y la respuesta fue nula, esto evidencia falta de interés de los empresarios para capacitarse y actualizarse con temas que pueden contribuir a elevar la rentabilidad de su negocio y que este a su vez se consolide y desarrolle a otras latitudes.

Con la finalidad de motivar a los empresarios se diseñó un programa de trabajo de la consultoría empresarial universitaria, que contempla la actualización y capacitación permanente de los consultores, estudiantes y empresarios, de temas de actualidad en áreas 
de administración, administración financiera, contabilidad, auditoria, costos, recursos humanos, mercadotecnia, comercio exterior, impuestos y legal, entre otros, con la finalidad de que cada uno de los planes de negocio, proyectos de inversión, así como las acciones y estrategias que se lleven a cabo en el proceso de la consultoría sean profesionales y fundamentadas en teorías, principios, investigaciones y casos exitosos en los negocios.

Una de las finalidades del programa a corto plazo, es que mediante el proceso de la consultoría, se logre fortalecer las finanzas de las microempresas del sector comercio de la zona metropolitana de Guadalajara, de aquellas empresas que en la actualidad se encuentran participando y desarrollando cada una de las acciones planteadas en el plan de acción, que a su vez debe estar supervisadas y monitoreadas de manera permanente, con la intención de que replique los resultados positivos en el futuro, y que sea un modelo exitoso que motive a otras empresas a participar en dicho programa y por ende lograr cada uno de los objetivos planteados, desde el PDI del Centro Universitario UTEG A.C.

El proyecto de la consultoría desarrollada en UTEG contempla reactivos que permiten detectar debilidades y necesidades de la microempresa a través de la aplicación de un pre diagnostico que evidencia los resultados de la estructura organizacional, contable, financiera y fiscal acercados a la realidad en tiempo efectivo.

(Cummins, 2007), establece cuatro perspectivas que según él permite identificar a través de la aplicación de un diagnostico empresarial los síntomas del comportamiento organizacional de una empresa:

1.-Direccionamiento.

2.- Áreas funcionales.

3.- Financiero.

4.- Externo.

Las cuatro perspectivas coadyuvan a identificar el comportamiento y dirección que ha tenido la empresa en los últimos años, así mismo facilita la evaluación del desempeño de las diferentes áreas funcionales, cual ha sido el manejo financiero de los recursos y el 
destino de los mismos, por ultimo permite valorar las tendencias políticas, económicas y sociales del entorno en la que se encuentra la empresa, para que esta pueda afrontar las diversas situaciones que se puedan suscitar en el coarto y mediano plazo.

En base a lo anterior y con la finalidad de obtener información confiable se diseñó un instrumento que permite identificar las características, fortalezas, oportunidades, debilidades y amenazas (FODA) de cada una de las empresas consultadas es el siguiente:

1. Nombre o razón social de la empresa

2. Personalidad jurídica: Persona Física o Persona Moral

3. Identifica en cual régimen fiscal tributa la empresa

4. La empresa cuenta con empleados y describir cada uno de los cargos y funciones

5. La empresa tiene un expediente fiscal

6. La empresa cuenta con sucursales y agencias

7. En la empresa realizan registros contables en pólizas de todas las operaciones monetarias y financieras

8. La empresa cuenta con un sistema de contabilidad que controle y emita información financiera

9. El sistema de contabilidad de la empresa en caso que lo tengan, que tipo de información financiera, contable, administrativa y fiscal genera, y con qué periodicidad

10. Sabe cuáles son las obligaciones fiscales de la empresa, su periodo de cálculo y pago

11. La empresa tiene definida su planeación estratégica (misión, visión, objetivos y organigrama)

12. La empresa cuenta con estructura organizacional

13. La empresa cuenta con presupuesto de ingresos y egresos

14. La empresa cuenta con un plan de negocios

15. Los tomadores de decisiones de qué manera lo hacen en las áreas administrativas, contables, financieras y fiscales

16. La empresa cuenta con asesores externos 
17. Conoce como se determinan los costos de su empresa

\section{Resultados}

Derivado de la aplicación directa del cuestionario a través de una entrevista que realizaron el equipo de consultores a los 30 empresarios, los resultados obtenidos evidencian una falta de cultura empresarial y se afirma que las decisiones que toman en los diferentes ámbitos empresariales son empíricos, no los sustentan a través de diagnósticos, informes o estados financieros, la mayoría de los empresarios respondieron en el mismo sentido destacando las siguientes afirmaciones de su propia voz:

1. No cuentan con un sistema de contabilidad que les permita registrar todas las operaciones financieras que se realizan en la entidad económica.

2. No se genera información contable y financiera que le permita al empresario o comerciante tomar decisiones en base a situaciones reales de su negocio.

3. Las decisiones administrativas, operativas y financieras las toman en base a hechos pasados y experiencias de éxito, sin que esto les garantice lograr los mismos resultados.

4. No cuentan con un proceso de asesoría externa profesional y empresarial en su negocio, con la finalidad de fortalecer la toma de decisiones.

5. No cuentan con suficientes recursos económicos que les permita contratar un asesor empresarial.

6. El costo que les representa para contratar un servicio de consultoría empresarial, lo perciben como un gasto innecesario y no como una inversión que les puede generar más recursos económicos y oportunidades de negocio.

7. No cuentan con controles internos en el área de crédito y cobranza, en los inventarios, en los procesos de cotización y compra de mercancías, entre otros.

8. No tienen definida su planeación estratégica y financiera.

9. Desconocen cuáles son sus obligaciones legales y fiscales tanto personales como de la empresa.

10. Las obligaciones fiscales no se cumplen de manera oportuna y los cálculos y la determinación de las contribuciones no están sustentadas en registros que cumplan con los ordenamientos jurídicos y fiscales vigentes. 
Se muestran varias graficas que permiten visualizar algunas debilidades y amenazas de la empresa, las cuales contribuyen a que los tomadores de decisiones lo hagan de manera errónea.

Figura No. 3

Identifica en cuál régimen fiscal tributa la empresa

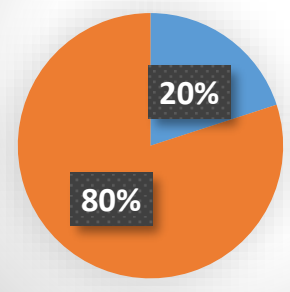

Fuente: Elaboración propia

Figura No. 5

\section{El sistema de contabilidad de la} empresa que tipo de información genera

\section{$3 \%$}

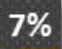

$0 \%$

$90 \%$

nContable,

administrativo y

Contable

Financiera y Fiscal

m Financiera y Fiscal

No genera

Fuente: Elaboración propia

Figura No. 7

Sabe cuáles son las obligaciones

fiscales de la empresa

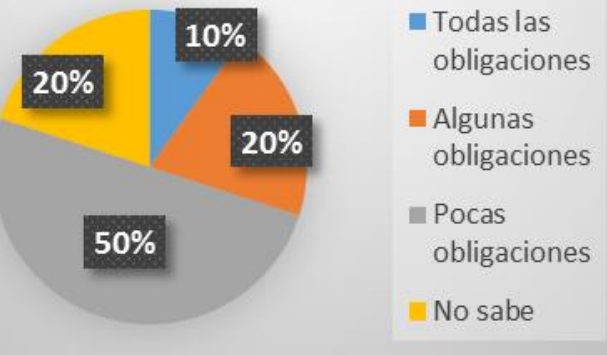

Figura No.4

La empresa cuenta con un sistema de contabilidad que controle y emita información financiera

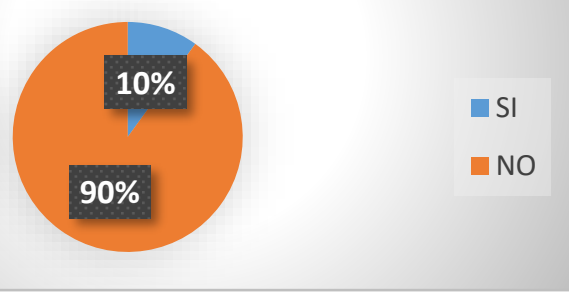

Fuente: Elaboración propia

Figura No. 6

La empresa tiene definida su planeación estratégica

$53 \%$

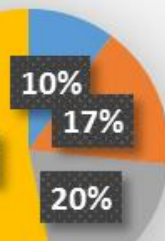

- Misión, Visión, Objetivos y Organigrama

- Misión, Visión y Objetivos
Fuente: Elaboración propia

Figura No. 8

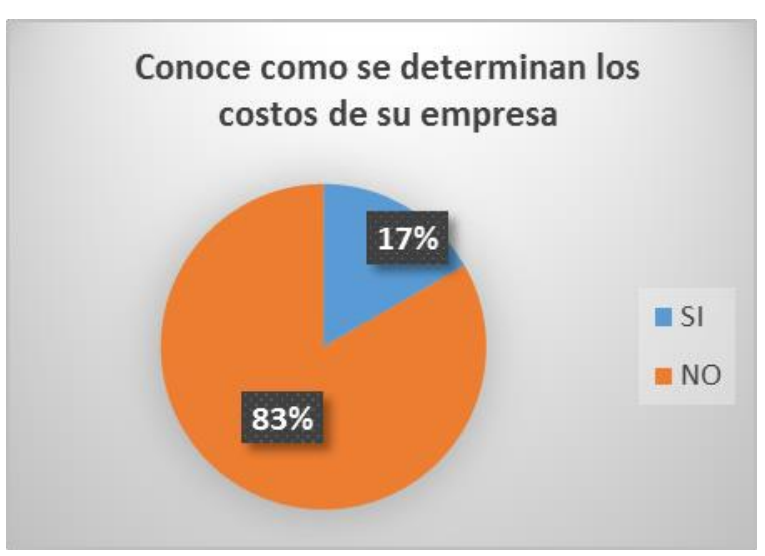


Fuente: Elaboración propia

Fuente: Elaboración propia

Es importante evidenciar la falta de una cultura empresarial y sobre todo el desconocimiento que tienen los microempresarios de cual es la situación económica, fiscal y financiera de su empresa, no saben si tienen utilidades o perdidas reales, ya que la información contable que generan es escasa e incompleta, no generan información confiable que les ayude a tomar decisiones acertadas en tiempo y forma para prevenir contingencias financieras, administrativas, legales y fiscales, que en ocasiones provocan el cierre la empresa, por falta de recursos financieros o endeudamiento con proveedores y con autoridades fiscales en sus tres niveles de gobierno (federal, estatal y municipal).

Una vez que se analizó la situación administrativa, contable y financiera actual de cada una de las microempresas consultadas se diseñó un plan de acción de acuerdo a la metodología establecida por el equipo consultor, el periodo de tiempo que llevo el proceso de la consultoría empresarial universitaria fue alrededor de cinco meses, llevándose a cabo capacitaciones con temas disciplinares en el ámbito empresarial, administrativo, contable, financiero y fiscal a empresarios, docentes y estudiantes de acuerdo a las debilidades y amenazas detectadas en cada una de las treinta empresas consultadas.

Lo anterior demuestra que la mayoría de las microempresas consultadas nos formulan estados financieros básicos, ya que carecen de un sistema de contabilidad que les permita registrar cada una de las operaciones financieras que realiza la entidad económica, siendo está técnica fundamental en los negocios.

El papel que juega la contabilidad en los procesos administrativos es fundamental lo afirma (Ramírez, 2013), está permite diseñar una planeación de acciones con el fin de alcanzar los objetivos deseados a un periodo determinado, además menciona que es necesaria por diferentes motivos entre los que destacan:

1.- Facilita la prevención de los cambios en el entorno, anticipándose a ellos es más fácil adaptarse a los nuevos requerimientos y necesidades económicas, sociales, políticas, entre otras. 
2.- Sirve para definir claramente los objetivos y decisiones de la organización.

3.- Promueve la comunicación, coordinación y cooperación de los diferentes actores que integran la empresa.

Uno de los instrumentos que son determinantes para lograr el éxito para el fortalecimiento de las finanzas de las empresas, según (Haro, 2008) son los estados financieros básicos como la Balanza de Comprobación, el Estado de Resultados Integral, el Estado de Posición Financiera, así como el Estado de Origen y Aplicación de Recursos, son una de las fuentes de información confiable más importantes que permiten tomar decisiones fundamentadas en hechos y situaciones reales a los empresarios y los tomadores de decisiones, además la formulación periódica (mensual) de dichos estados financieros son obligatorios de acuerdo al artículo 33 del Código de Comercio, el artículo 28 del Código Fiscal de la Federación y el articulo 33 del Reglamento del Código Fiscal de la Federación, todos vigentes.

\section{Conclusiones}

A pesar de los avances tecnológicos, científicos y económicos que se han venido suscitando en los últimos cincuenta años, los microempresarios carecen de preparación técnica y científica que les permita tomar decisiones acertadas en la conducción y administración de su negocio, la falta de capacitación permanente en temas sociales y económicos, así como de las diferentes áreas funcionales de la organización, no contribuyen a fortalecer las finanzas de su empresa.

Fueron alrededor de siete meses el tiempo que duro el proceso de la implementación del programa de consultoría empresarial universitaria, como ya se menciono fue difícil convencer a los empresarios de las bondades y beneficios que el programa les iba a generar a través de la intervención y asesoría del equipo consultores en temas contables, administrativos, de costos, de auditoria y fiscales, todos del alguna manera contribuyen a fortalecer las finanzas de su empresa o negocio, y además este proceso de acompañamiento es gratuito, no les representa costo alguno, lo único que se les pidió es participación responsable y colaborativa en todas las etapas de la consultoría. 
En el transcurso de los siete meses se inició con treinta empresas a las cuales se les aplico el diagnostico FODA ya mencionado, en el proceso de sensibilización, capacitación y actualización de los empresarios y el equipo consultor, alrededor de diez empresarios abortaron el proceso, dejaron de asistir, y en la etapa de la intervención directa a la empresa a través del plan de trabajo implementado de manera conjunta entre los empresarios y consultores, dejaron de acudir a las reuniones de trabajo nueve empresarios, dejando evidente la falta de interés en el trabajo colaborativo.

Respecto al último grupo de microempresarios, se tuvo la experiencia con el programa de Hecho por mujeres en colaboración con el gobierno municipal de Guadalajara, también es evidente que el interés de su participación es por un motivo.

Para obtener Capital semilla que el gobierno les proporcionará para poder abrir el negocio (alrededor de \$100,000.00 cien mil pesos mexicanos por empresa).

De éste último grupo de microempresarios comenzamos el proceso con treinta equipos de trabajo y lamentablemente durante el tiempo que se llevó a cabo la consultoría empresarial desertaron veinte, lo que quiere decir que únicamente concluyeron de manera satisfactoria diez microempresas, a las cuales se les elabora el informe final de la consultoría realizada.

Derivado de los resultados obtenidos, el equipo consultor concluyo que es un reto para las Instituciones de Educación Superior (IES), como lo es el Centro Universitario UTEG A.C., redoblar esfuerzos de manera conjunta con el Gobierno y Cámaras o Agrupaciones Empresariales para sensibilizar a los microempresarios de la importancia y beneficios que tiene un proceso de consultoría empresarial en su negocio, además que la capacitación y actualización disciplinar, técnica, científica y empresarial debe estar presente en todo momento, de tal manera que les ayude a tomar mejores decisiones a corto, mediano y largo plazo, trayendo como consecuencia natural un mejor nivel de vida para la sociedad. 
La finalidad de la metodología empleada en el programa de consultoría empresaria de UTEG como ya se menciono es fortalecer las finanzas de la empresa u organización, de esta manera el ciclo de vida se extiende y a su vez la unidad económica se consolida, crece y desarrolla.

\section{Bibliografía}

Augusto, Carlos (1994) Del desarrollo organizacional, Editorial Trillas, México.

Centro Universitario UTEG A.C. (2014). Plan de Desarrollo Institucional. 10 de Agosto de 2016, de Centro Universitario UTEG A.C. Sitio web: http://www.uteg.edu.mx/noticias/2014/05/08/consulta_el_pdi_2025

Cohen, William (2003) Cómo ser un consultor exitoso, Editorial Norma, Bogotá, Colombia. Jalisco.

Cummins, T. y Worley, C. (2007). Desarrollo Organizacional y Cambio. Editorial Thomson. México.

Furnham. A. (2001) Psicología Organizacional "El comportamiento del individuo en las organizaciones". Editorial Oxford. México.

H. Cámara de Diputados. (2016). Código de Comercio. 10 de julio de 2016, de Diario Oficial dela Federación Sitio web: http://www.sat.gob.mx/informacion_fiscal/normatividad/Paginas/2016/leyes_2016.a spx

H. Cámara de Diputados. (2016). Código Fiscal de la Federación. 14 de julio 2016, de Diario Oficial de la Federación Sitio web: http://www.sat.gob.mx/informacion_fiscal/normatividad/Paginas/2016/leyes_2016.a spx

Haro, Miguel y Silva, Biviana. (2008). Introducción a la Contabilidad y su Fundamentos Legales. Editorial Umbral. México.

Hernández Jorge A. (2011). Desarrollo Organizacional "Enfoque Latinoamericano", Editorial Pearson. México, DF

Kubr Milan. (2015). La Consultoría de Empresas "Guía para la profesión”. Limusa, 3ra. Ed. México. 
OCDE, CNF, CEPAL, (2014). Perspectivas económicas de América Latina 2014, Logística y competitividad para el desarrollo. CEPAL-OCDE, México.

Ramírez, David (2013). Contabilidad Administrativa "Un enfoque estratégico para competir”. Editorial Mc Graw Hill. México.

Secretaría de Economia. (2009). ACUERDO por el que se establece la estratificación de las micro, pequeñas y medianas empresas. 20 de Julio 2016, de Diario Oficial de la Federación Sitio web: http://dof.gob.mx/nota_detalle_popup.php?codigo=5096849

Secretaría de Hacienda y Crédito Público. (2014). Reglamento del Código Fiscal de la Federación. 28 de Agosto de 2016, de Diario Oficial de la Federación Sitio web: http://www.sat.gob.mx/informacion_fiscal/normatividad/Paginas/reglamentos_legisl acion.aspx 